\title{
Study of Diffusion Instability in Ternary Gas Mixtures under Nonisothermal Conditions
}

\author{
Vladimir Kossov $^{1, \mathrm{a}}$, Yuri Zhavrin ${ }^{2}$ and Olga Fedorenko ${ }^{2}$ \\ ${ }^{1}$ Abai Kazakh National Pedagogical University, Almaty 050010, Kazakhstan \\ ${ }^{2}$ Research Institute of Experimental and Theoretical Physics, Al-Farabi Kazakh National University, Almaty 050040, \\ Kazakhstan
}

\begin{abstract}
The main objective of this article is to investigate the effect of heating (from below or above) on stability of mechanical equilibrium in binary and ternary gas mixtures. Three series of experiments on instabilities in are reported gaseous diffusion. In one series occurrence of convective flows under diffusive mixing are investigated in the systems $\mathrm{N}_{2} \mathrm{O}-\mathrm{CO}_{2}$ and $\mathrm{CO}_{2}-\mathrm{N}_{2} \mathrm{O}$ heated from below. The temperature difference when the transition between the diffusion and the convective processes happen is higher at the bottom arrangement of $\mathrm{N}_{2} \mathrm{O}$. In the second series the influence of temperature difference are studied in the mixture $0.279 \mathrm{C}_{3} \mathrm{H}_{8}+0.721 \mathrm{CO}_{2}-\mathrm{CO}_{2}$. The rate of convective mass transfer increases sufficiently. In the third series the convective mass transfer is examined in the system $0.4736 \mathrm{He}+0.5264 \mathrm{Ar}-\mathrm{N}_{2}$ heated from above. The experimental results reveal that the intensity of unstable process decreases until $420.0 \mathrm{~K}$ reaching a minimum value. Linear stability theory is applied to determine the boundary of transition from the state of diffusion to the region of convection. The results of numerical study are in good agreement with the experiments.
\end{abstract}

\section{Introduction}

One of the features of multicomponent mixing is the occurrence of the gravitational concentration convection under diffusion. Sometimes this peculiarity is called as an anomalous diffusion. The effect anomality is that the instability of mechanical equilibrium with the further formation of convective flows happens under diffusion.

Available experimental data indicates that the phenomenon of diffusion instability (double-diffusive convection) have been studied in detail in liquids, in a porous medium saturated with nanofluid today [1-7]. Researches connected with the study of mechanical equilibrium under isothermal and nonisothermal conditions for gases are insufficient [8-11].

There are two reasons of convective flows onset under temperature gradient it is nonuniformity of temperature and concentration as well as two dissipative mechanisms, i.e. heat conductivity and diffusion. It results in qualitative novel effects. Phenomenon becomes more complicated due to the cross-effects, i.e. thermodiffusion and diffusion heat conductivity $[7,12]$.

Transition of the system from the stable state to the unstable one is characterized essentially by the diffusion coefficients difference of components. The problem is considerably complicated when the unstable diffusion process occurs in the nonisothermal conditions. On the one hand, appearance of the temperature gradient under certain conditions may intensify the unstable process [7]. On the other hand, the raise of temperature results in the certain stabilization of unstable process [9].

\section{Experimental}

To research the diffusion instability under nonisothermal condition, we used a two-flask method where the analysis of gas mixtures before and after diffusion was carried out by the interferometric and chromatographic methods. The method of the two-flask diffusion apparatus is one of the classical method used to measure the coefficient of interdiffusion, self-diffusion and thermodiffusion in the wide area of parameters (pressure, temperature, concentration).

To carry out the research we chose the following gaseous systems $\mathrm{N}_{2} \mathrm{O}-\mathrm{CO}_{2}, 0.279 \mathrm{C}_{3} \mathrm{H}_{8}+0.721 \mathrm{CO}_{2}-$ $\mathrm{CO}_{2}$ and $0.4736 \mathrm{He}+0.5264 \mathrm{Ar}-\mathrm{N}_{2}$ where the concentrations of components are given in the mole fractions. The composition of binary mixture was that the density of mixture, which was located in the upper flask, would be always less than the density of pure gas placed in the lower part of apparatus at any temperature of experiment.

\footnotetext{
a Corresponding author: kosov_vlad_nik@list.ru
} 


\subsection{Apparatus and procedure}

\subsubsection{Apparatus}

The experimental setup consisted of two parts, as shown schematically in Figure 1. The first one was a gas preparation unit. It consists of two pressure gages (12) to measure the pressure in the flasks, a tank (13) to equalize the pressure in the flasks of the diffusion apparatus, and a set of the needle valves (stopcocks) (1-10) to fill the setup flasks with appropriate gas mixtures from the cylinders (20) and (21). The pressure gages were supplied with the specially prepared membrane dividers (11).

The second part of the setup was the diffusion apparatus with the upper and lower flasks of volume $62.0 \cdot 10^{-6} \mathrm{~m}^{3}$. The flasks were connected through a cylindrical diffusion channel of geometrical sizes: the length and the diameter of diffusion channel were $4.00 \cdot 10^{-3}$ and $63.10 \cdot 10^{-3} \mathrm{~m}$, respectively.

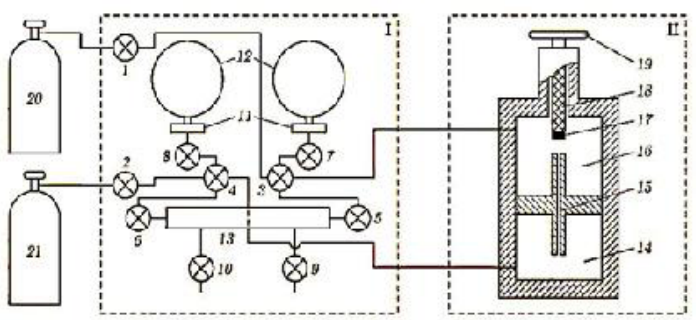

Figure 1. Block diagram of the installation of the two-flask method. The gas preparation block and the two-flask diffusion setup are indicated by I and II, respectively; valves (1-8); valve connected to the backing vacuum pump (9); valve connected to the interferometer or chromatograph (10); membrane dividers (11); reference pressure gages (12); equalizing tank (13); bottom flask (14); diffusion channel (15); top flask (16); fluoroplastic pellet (17); rod (18); handwheel (19); gas holders (20) and (21).

The experiments lasted 1200 and $3600 \mathrm{~s}$ that made it possible to obtain the full information about the character of the examined mass transfer.

While filling, and for monitoring the pressure during the experiments there have been used in the reference pressure gauges to the corresponding measurement range to an accuracy class of 0.4 .

The diffusion apparatus was placed directly in the thermostatic bath by means of which the temperature could be held constant in one of the flask to an accuracy of $\pm 0.1 \mathrm{~K}$.

\subsubsection{Experimental procedure}

The experiments were carried out using a method proposed in works $[9,13,14]$.

The experimental procedure on the installation was as following. As soon as the upper flask (16) and the lower one (14) of the apparatus were washed by the gases studied from the cylinders (20) and (21), the both flasks were filled with the gas up to the experimental pressure. The fluoroplastic pellet (17) ensured leakproofness while filling the flasks. The diffusion channel (15) was opened by upraising a rod (18) using a handwheel (19), and simultaneously the time corresponding to the mixing process beginning was registered. The initial composition of binary mixture was analyzed by an interferometer with the resultant error not exceeding $0.1 \%$ and by a chromatograph with the error any more than $0.3 \%$ after the diffusion process.

\subsection{Experimental results}

In first series of experiments the study of the temperature gradient influence was carried with gases, the densities of which are almost the same under normal conditions. We began with a study in the system $\mathrm{N}_{2} \mathrm{O}-\mathrm{CO}_{2}$. Temperature of the upper flask was equal to $283.0 \mathrm{~K}$ and that of the lower one ranged from $303.0 \mathrm{~K}$ to $363.0 \mathrm{~K}$. The experiment lasted $1200 \mathrm{~s}$ and the excess pressure was $p=1.07 \mathrm{MPa}$. The experimental results are shown in Figs. 2 and 3 as the dependence of non-dimensional parameter $\alpha=\frac{c_{\text {exp }}}{c_{\text {theor }}}$ on the temperature difference $\Delta t$ of flasks. Lines approximating the experimental results are presented on the same figures too.

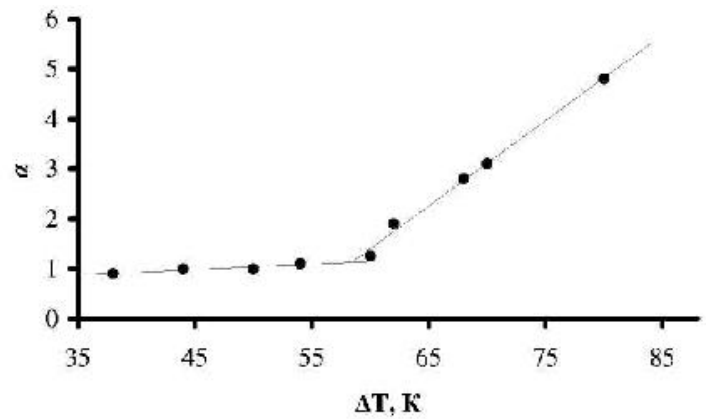

Figure 2. Temperature difference of the parameter $\alpha\left(\mathrm{CO}_{2}\right.$ is located above, $\mathrm{N}_{2} \mathrm{O}$ is placed below).

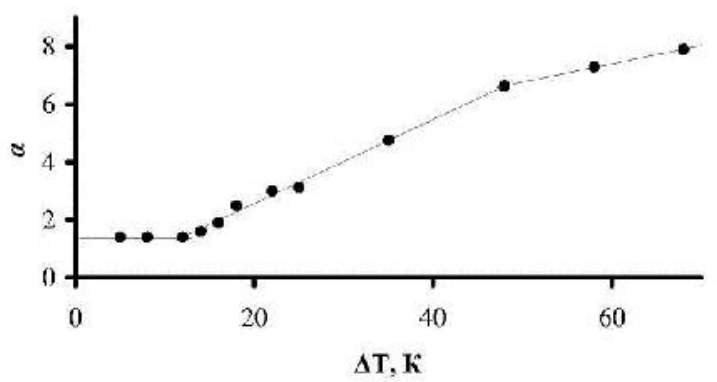

Figure 3. Temperature difference of the parameter $\alpha\left(\mathrm{N}_{2} \mathrm{O}\right.$ is located above, $\mathrm{CO}_{2}$ is placed below).

In the system nitrous oxide-carbon dioxide the density gradient is equal to zero in case of idealness of diffusing components. Therefore, the diffusion Rayleigh number $\mathrm{Ra}_{c}=0$ and temperature gradient behave due to the heating from the bottom, i.e. we can find the heat Rayleigh number $\mathrm{Ra}_{\square}^{c r}$ characterizing the break of diffusion process under determined temperature difference $\Delta \square_{c r}$. As the densities of investigated gases are practically the same then it would be expected that the overturn of system could not effect on the value of critical temperature difference, however, the experiment shows the significant distinction that is presented on Figs. 
2 and 3. Idealness of examined gases must be the reason of this result.

Thus, the temperature gradient influences on the nature of mixing process in binary mixtures. Convective mixing process being due to the density gradient transfers into the diffusion one under defined value of temperature gradient directed opposite. Also, we acknowledge the fact that diffusion instability occurs in equimolar gas system at a certain temperature difference at the ends of the diffusion path. Critical parameters of the mode change depend on gases arrangement relative to each other. The difference between these parameters can be due to the appearance of non-ideal properties of components.

To determine the reasons for the influence of the gas orientation relative to the diffusion channel on the stability of the diffusion process in the presence of a temperature gradient, we have compiled a system consisting of propane and carbon dioxide. In the experiment, binary gas mixture consisting of $27.94 \%$ $\mathrm{C}_{3} \mathrm{H}_{8}$ and $72.06 \% \mathrm{CO}_{2}$ was placed in the upper flask of diffusion apparatus and pure carbon dioxide was located at the bottom of one. Experiments were carried out on the diffusion installation and according to the procedure as described above. The total pressure was $0.6 \mathrm{MPa}$, and the temperature of the upper flask of diffusion apparatus was maintained constant and equaled to $283.00 \mathrm{~K}$, temperature at the bottom flask varied from $303.0 \mathrm{~K}$ to $363.0 \mathrm{~K}$. Time of the experiment was $20 \mathrm{~min}$. The results of experimental study are shown in Fig. 4.

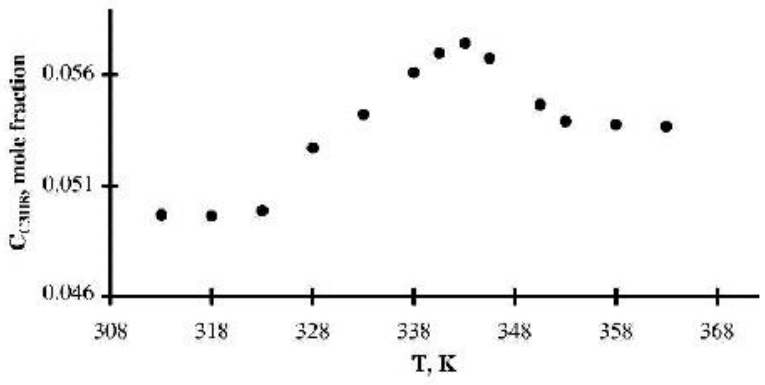

Figure 3. Temperature dependence of the passed propane concentration at the bottom flask of diffusion apparatus.

As can be seen from the data, mixing process is unstable when changing the temperature of the bottom flask of diffusion apparatus. To a temperature of $323.0 \mathrm{~K}$ change in the concentration of propane is not observed. This may occur when the mixing process is diffusion one. With increasing of temperature, the concentration of propane in the bottom flask increases due to the fact that a cold region has a higher density, so propane "sinks" to the bottom flask, causing a convective flow. This occurs before the temperature of $343.0 \mathrm{~K}$. With further increasing of the temperature, a decrease of concentration is observed that can be explained by the mutual influence of the temperature gradient and the concentration gradient. From the temperature of $350.0 \mathrm{~K}$ change in the concentration of the diffused propane remained virtually unchanged with increasing of the temperature, due to the stabilization of the convective mixing process.

Thus, the change in density due to temperature changes can cause unstable diffusive mixing process.
Mutual influence of temperature and concentration gradients causes convection currents of varying intensity.

In third series of experiments the study was conducted on the system $0.4736 \mathrm{He}+0.5264 \mathrm{Ar}-\mathrm{N}_{2}$ where the concentrations of components are given in the mole fractions. The composition of binary mixture was that the density of mixture that was located in the upper flask would be always less than the density of pure gas placed in the lower part of apparatus at any temperature of experiment. The essence of experiment was in the following. Binary mixture that revealed unstable behaviour under diffusion into the pure component at the temperature $T=278.0 \mathrm{~K}$ was chosen. Thereafter, the temperature of only upper flask was changed until the maximum value $T=473.0 \mathrm{~K}$ from one experiment to another one. The temperature of lower flask was maintained constant and was equal to $T=298.0 \mathrm{~K}$. At the end of each experiment, the compound of gas mixtures was analyzed from both flasks of apparatus. The results of experiment were compared with the data calculated by the Stephan-Maxwell approach with the assumption that the given system was stable at the mean temperature $T=\langle T\rangle=0.5\left(T_{1}+T_{2}\right)$ (i.e. the concentration convection was absent). Figure 4 shows the behaviour of concentrations of diffused components subject to the temperature of hot vessel. The experiments lasted an hour in all cases.

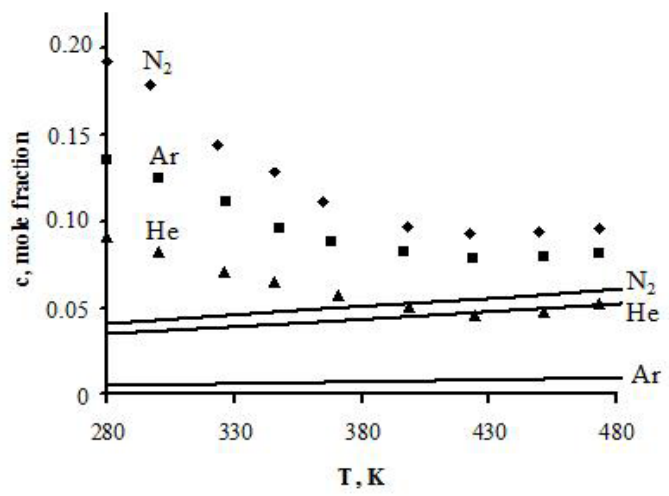

Figure 4. Concentration of the diffused components vs. the temperature. Symbols denote the experimental data. Full lines are the calculation.

As it is seen in Fig. 1, the intensity of unstable process drops with the increasing of the pressure in the upper flask and is at its maximum at the temperature close to $420.0 \mathrm{~K}$. The transfer of argon and nitrogen is well over than of helium that it is typical of the unstable diffusion. Further increase of temperature results in the concentration growth of helium (coincidence of experimental and calculated data is observed) at the practically constant concentrations of other components. From here on the molecular transfer seems to be prevalent over the convective one, though the intensity stabilization of the unstable process of each component is possible at the different temperatures.

Stabilization of unstable process intensity can be explained by analogy with [8,9] which considered isothermal system. Let us suppose that, in our case, at a certain average value of the temperature 
$T=\langle T\rangle=0.5\left(T_{1}+T_{2}\right)$ diffusion process will also be unstable. With increasing temperature conditionally stratified region of the gas mixture due to increasing diffusion rates of components starts to "diffuse" along the diffusion channel, and the system tends to a stable state. For stable process a monotonic increase in the concentrations of all three components with increasing temperature (calculation is performed according to the Stefan-Maxwell scheme under the assumption that the system is stable at $T=\langle T\rangle$ ) is observed. Note the following feature. Under diffusion instability in an inhomogeneous temperature field mass transfer stabilization of components occurs at different temperatures. It is $420.0 \mathrm{~K}$ for the helium under these experimental conditions, (agreement between the experimental points with the calculated data in a stable mode of diffusion is shown in Fig. 4). The nitrogen temperature is $800 \mathrm{~K}$ (data obtained by comparing the calculated and experimental values by extrapolation to a temperature region above $500 \mathrm{~K}$ ). Regarding argon, in a given time interval in a temperature of $500 \mathrm{~K}$ and above, in the system there is a "diffusion gate" [15] which disappears during much more diffusion times. Therefore the results comparison failed.

In conclusion, we note the difficulty in setting up the experiment to study the unstable diffusion process in the presence of temperature gradient in ternary gas mixtures, as well as determining the boundaries of stable diffusion. Therefore there is a necessity to create reliable methods for predicting the behavior of systems in a wide range of thermodynamic parameters.

\section{Mathematical model}

In this section, we consider a stability theory to define the arrangement of transition boundaries between the diffusion and the concentration gravitation convection.

\subsection{Model of diffusion instability in binary mixtures}

The macroscopic motion of a binary gas mixture in a temperature field will be described in terms of the stability theory $[7,10,16]$. In this case, the general set of equations of fluid dynamics consists of the Navier-Stokes equation of motion, the continuity equation, and the heat and mass transfer equations:

$$
\begin{gathered}
\rho\left[\frac{\partial \mathbf{u}}{\partial t}+(\mathbf{u} \nabla) \mathbf{u}\right]=-\nabla p+\eta \nabla^{2} \mathbf{u}+\rho \mathbf{g} \\
\rho T\left(\frac{\partial s}{\partial t}+\mathbf{u} \nabla s\right)=-\operatorname{div} \mathbf{q}+\mu \operatorname{divj} \\
\rho\left(\frac{\partial c}{\partial t}+\mathbf{v} \nabla \mathbf{c}\right)=-\operatorname{divj} \\
\frac{\partial \rho}{\partial t}+\operatorname{div}(\rho \mathbf{v})=0
\end{gathered}
$$

where $\alpha_{p}=\frac{m_{2}-m_{1}}{c_{1} m_{1}+c_{2} m_{2}}$ and $\mu=\frac{\mu_{1}}{m_{1}}+\frac{\mu_{2}}{m_{2}}$.

The flow densities $\mathbf{j}$ and $\mathbf{q}$ from the equation (1) can be expressed as the gradients of temperature, concentration, and pressure. If considerable pressure gradients are absent in the given gas mixture, we shall have

$$
\begin{gathered}
\mathbf{j}=-\rho D_{12}\left(\nabla c+\frac{k_{T}}{T} \nabla T\right) \\
\mathbf{q}=-\chi \nabla T+\left[\mu-\left(\frac{\partial \mu}{\partial T}\right)_{c, p}+k_{T}\left(\frac{\partial \mu}{\partial c}\right)_{T, p}\right] \mathbf{j}
\end{gathered}
$$

The method of small perturbation [7] has been used by solution the system of Equation (1). Taking into account that the difference between perturbation of the average $v$ and the weight-average $u$ velocities will be inconsiderable, and then the final system of equations for perturbation values in dimensionless quantities takes the form:

$$
\begin{gathered}
\frac{\partial \mathbf{u}}{\partial t}=-\nabla p+\nabla^{2} \mathbf{u}+\left(\operatorname{Ra}_{c} T+\operatorname{Ra}_{T} c\right) \boldsymbol{\gamma} \\
P \frac{\partial T}{\partial t}-(\mathbf{u} \boldsymbol{\gamma})=(1+a) \nabla^{2} T+\frac{a}{b} \nabla^{2} c \\
P_{d} \frac{\partial c}{\partial t}-(\mathbf{u} \boldsymbol{\gamma})=\nabla^{2} c+b \nabla^{2} T
\end{gathered}
$$

$$
\operatorname{div} \mathbf{u}=0
$$

where $r$ is the characteristic distance measurement scale; $r^{2} / v$ stands for the time; $\chi / r$ stands for the rate; $A r$ stands for the temperature; $B \chi r / D_{12}$ stands for the concentration ( $A$ is determined from the expression $\nabla T_{0}=-A \gamma ; B$ from $\left.\nabla c_{0}=-B \gamma\right)$; with $\rho_{0} v \chi / r^{2}$ being the pressure; $\mathrm{Ra}_{c}=\frac{g \beta_{1} A r^{4}}{v \chi}$ and $\mathrm{Ra}_{T}=\frac{g \beta_{2} B r^{4}}{v D_{12}}$ are the Rayleigh diffusion and heat numbers; $v=\eta / \rho_{0}$; $P=v / \chi ; \quad P_{d}=v / D_{12} ; \quad a=\alpha^{2} N D_{12} / \chi \quad$ and $b=\alpha D_{12} A / \chi B$ are the parameters characterizing thermal diffusion and diffusion heat conductivity; $\alpha=k_{T} / T$; $N=\left[\frac{T}{C_{p}}\left(\frac{\partial \mu}{\partial c}\right)_{T, p}\right]_{0}$ is a thermodynamic parameter (the values in square brackets are taken at average values of the temperature and the concentration). If the temperature gradient is small, the cross effects are assumed to be insignificant, i.e. $a=b=0$.

It is necessary to define exactly the boundary conditions for the solution of the system of Eq. (3). Therefore we have considered the unstable diffusion mixing problem in a finite flat vertical channel. It is 
assumed that the perturbations are plane-parallel, are directed along $z$-axis, and depend only on one coordinate $x$. The boundary conditions suggest that the velocity and perturbations of the concentration and the temperature become zero in the vertical planes limiting the layer of the gas mixture:

$$
u=c=T=0, \quad x= \pm 1
$$

The equation system (3) is solved by the method given in []. As the result, we determine the boundary line of monotonic perturbations in the form

$$
\mathrm{Ra}_{c}+\mathrm{Ra}_{T}=\pi^{4}
$$

This equation in the coordinates $\left(\mathrm{Ra}_{c}, \mathrm{Ra}_{T}\right)$ gives a straight line MM, dividing the region of molecular transport and the region of the diffusion instability. Figure 5 shows the location of the neutral line of monotonic instability for the system $\mathrm{N}_{2} \mathrm{O}-\mathrm{CO}_{2}$. The region that lies below the line $\mathrm{MM}$ corresponds to diffusion process.

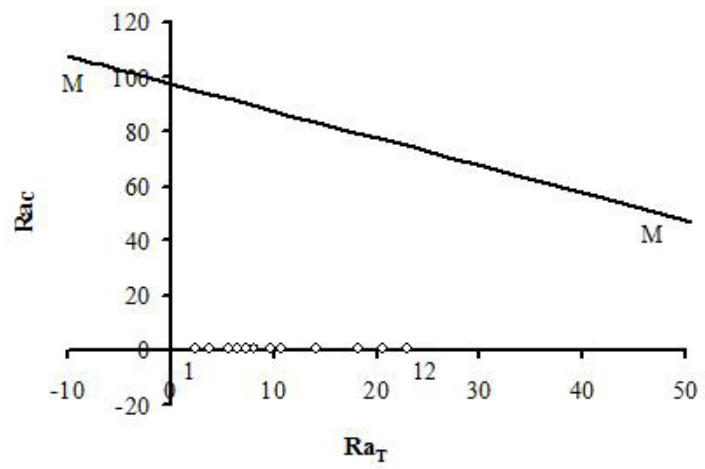

Figure 5. Line of monotonic perturbations $\mathrm{MM}$ and the experimental data for the system $\mathrm{N}_{2} \mathrm{O}-\mathrm{CO}_{2}$ on the plane $\left(\mathrm{Ra}_{c}\right.$, $\mathrm{Ra}_{T}$ ). Symbols $\circ$ correspond to data presented on Fig. 3. The calculation is carried out at the temperature difference value: $\Delta \mathrm{T}=5$ (1), 8 (2), 12 (3), 14 (4), 16 (5), 18 (6), 22 (7), 25 (8), 35 (9), 48 (10), 58 (11), 68 (12) K.

To compare the approach presented in this paragraph with experimental data is shown in Figs. 2 and 3 we present them in the form of partial Rayleigh number. The partial Rayleigh numbers in accordance with (3) can be written as follows

$$
R_{c}=\frac{g r^{4} n \Delta m \Delta c}{\rho v D_{12} L} \quad \text { and } \quad R_{T}=\frac{g r^{4} n \frac{\bar{m}}{\bar{T}} \Delta T}{\rho v \chi L}
$$

Here $\quad n=\frac{p}{k \bar{T}}, \Delta m=m_{1}-m_{2} \quad$ is the difference between the molecular masses of the first and second components, $\Delta c=c_{I}-c_{I I}$ is the difference between the concentrations of the selected component in the top and bottom flasks, $\Delta T=T_{I}-T_{I I}$ is the difference between the temperatures of the diffusion apparatus flasks, and $\bar{m}=c_{1} m_{1}+c_{2} m_{2}$.
Figure 5 shows the experimental data in terms of the Rayleigh numbers for the system $\mathrm{N}_{2} \mathrm{O}-\mathrm{CO}_{2}$ obtained at constant pressure and by varying the temperature gradient (difference).

On Fig. 5 points displaying the experiment are situated in the area of stability, but with temperature difference rise tend to the region of unstable diffusion. The results don't reveal the qualitative agreement with the experimental data shown in Fig. 3. The reason of divergency of results is that we didn't take into consideration real properties of mixing components.

\subsection{Model of diffusion instability in ternary mixtures}

The state of ideal ternary gas mixture within the bounds of the hydrodynamic approximation (pressure diffusion is negligible) is described by the conservation equations [7]:

$$
\begin{gathered}
\frac{\partial \mathbf{u}}{\partial t}+(\mathbf{u} \nabla \mathbf{u})=-\frac{1}{\rho} \nabla p+v \Delta \mathbf{u}+g \boldsymbol{\gamma} \\
\operatorname{div}(\rho \mathbf{u})=0 \\
\frac{\partial c_{i}}{\partial t}+(\mathbf{u} \nabla) c_{i}=\operatorname{div} \mathbf{j}_{i} \\
\mathbf{j}_{i}=\frac{n^{2}}{\rho} \sum_{j=1}^{v} m_{i} m_{j} D_{i j} \nabla c_{j}-D_{i}^{T} \nabla \ln T \\
\frac{\partial T}{\partial t}+(\mathbf{u} \nabla) T=\operatorname{divq}, \quad \mathbf{q}=-\chi_{\infty} \nabla T
\end{gathered}
$$

where $\mathbf{u}$ is the convection velocity; $\mathrm{p}$ is the pressure; $v$ and $\rho$ are the density and the kinematic viscosity of mixture; $c_{i}, m_{i}$ are the concentration and the molecule mass of $i$-th component; $T$ is the temperature; $D_{i j}, D^{T}$, $\chi_{\infty}$ are the coefficients of diffusion, thermal diffusion and heat conduction of mixture in the stationary state; $\gamma$ is the unit vector directed straight up. Simplify problem. Let us consider a horizontal layer limited by the parallel plane and the plane distance is equal to $d$ ( $z$-axis is directed straight up but $x$-axis and $y$-axis identify the plane perpendicular to the $z$-axis). Convective supplements are considered to be small against the density perturbations introduced by them as compared to $\rho_{0}$. Cross effects - thermal diffusion and diffusion heat conduction - are negligible. State equation can be written as

$$
\begin{aligned}
& \rho=\rho_{0}\left(1+\beta T^{\prime}+\sum_{i=1}^{3} \beta_{i} c_{i}^{\prime}\right), \sum_{i=1}^{3} c_{i}=1 \\
& \beta=-\frac{1}{\rho_{0}}\left(\frac{\partial \rho}{\partial T}\right), \beta_{i}=-\frac{1}{\rho_{0}}\left(\frac{\partial \rho}{\partial c_{i}}\right)
\end{aligned}
$$

where $\rho_{0}$ is a mixture density corresponding to the average values of temperature and concentration. Substituting Eq. (2) and perturbed values into Eq. (1), and 
after linearization within the Boussinesq approximation by analogy with [7] (the primes at the perturbed values being omitted) we have

$$
\begin{gathered}
\frac{\partial c_{i}}{\partial t}+\mathbf{u} \nabla c_{i}=D_{i i}^{*} \nabla^{2} c_{i}+D_{i j}^{*} \nabla^{2} c_{j} \\
\frac{\partial T}{\partial t}+\mathbf{u} \nabla T=a \nabla^{2} T \\
\frac{\partial \mathbf{u}}{\partial t}+(\mathbf{u} \nabla) \mathbf{u}=-\frac{1}{\rho_{0}} \nabla P+v \Delta \mathbf{u}+g\left(\beta T+\beta_{1}^{*} c_{1}+\beta_{2} c_{2}\right) \boldsymbol{\gamma}
\end{gathered}
$$

where $\beta_{i}^{*}=\beta_{i}-\beta_{3}, a$ is a temperature diffusivity coefficient of the mixture. To obtain a system of equations in dimensionless form, we introduce the scale unit: $d$ is a distance; $d^{2} / D_{11}^{*}$ is a time; $D_{11}^{*} / d$ is a velocity; $\delta c_{i} / d$ is a concentration gradient and $\delta T / d$ is a temperature. Using a stream function for the perturbations of velocity, we have

$$
\begin{gathered}
\frac{\partial c_{1}}{\partial t}-\nabla^{2} c_{1}-A \nabla^{2} c_{2}=-\frac{\partial \psi}{\partial x} \\
\frac{\partial c_{2}}{\partial t}-B \nabla^{2} c_{1}-\tau_{22} \nabla^{2} c_{2}=-\frac{\partial \psi}{\partial x} \\
\frac{\partial T}{\partial t}-\mathrm{Le}_{1} \nabla^{2} T=-\frac{\partial \psi}{\partial x} \\
\left(\frac{1}{\operatorname{Pr}_{1}} \frac{\partial}{\partial t}-\nabla^{2}\right) \nabla^{2} \psi=\operatorname{Ra}_{1} \frac{\partial c_{1}}{\partial x}+\tau_{22} \operatorname{Ra}_{2} \frac{\partial c_{2}}{\partial x}+\operatorname{Le}_{1} \operatorname{Ra} \frac{\partial T}{\partial x}
\end{gathered}
$$

where $\quad \mathrm{Ra}=g \beta \delta T d^{3} / v a, \quad \mathrm{Ra}_{i}=g \beta_{i}^{*} \delta c_{i} d^{3} / v D_{i i}$ are thermal and concentration Rayleigh numbers; $\beta_{i}^{*}=\beta_{i}-\beta_{3} ; \mathrm{a}$ is the thermal diffusivity of mixture; $\operatorname{Pr}_{i}=v / D_{i i}$ is the diffusion Prandtl number; $\mathrm{Le}_{i}=a / D_{i i}$ is the Lewis number; $\tau_{i j}=D_{i j} / D_{i i}$ denotes the parameters, which determine the relationship between the diffusion coefficients; $A=\tau_{i j} \delta c_{j} / \delta c_{i}, B=\tau_{j i} \delta c_{i} / \delta c_{j} ; \psi$ is the stream function for the perturbed velocity.

The boundary conditions assume that the flat layer is surrounded by the heat-conducting area then the heat conduction equation for the perturbed temperature is equal to

$$
\Delta T_{m}=0
$$

The following conditions for the velocity, temperature and components concentrations are satisfied at the stratum boundaries

$$
\mathbf{u}=0, T=T_{m}, \chi \frac{\partial T}{\partial n}=\chi_{m} \frac{\partial T_{m}}{\partial n}, \frac{\partial T}{\partial n}=\frac{\partial c_{1}}{\partial n}=\frac{\partial c_{2}}{\partial n}
$$

It is assumed that the solution of the set (9) has the form

$$
\begin{gathered}
\psi=\psi_{0} \sin (\pi l x) \sin (\pi n z) \sin (\pi m y) \exp (-\lambda t) \\
\left\{\begin{array}{l}
c_{1} \\
c_{2} \\
T
\end{array}\right\}=\left\{\begin{array}{l}
c_{1}^{0} \\
c_{2}^{0} \\
T^{0}
\end{array}\right\} \cos (\pi l x) \sin (\pi n z) \sin (\pi m y) \exp (-\lambda t)
\end{gathered}
$$

where $l, m, n$ are the modes of perturbations; $\lambda$ is the perturbation decrement. Substituting (12) into (9) and excluding successively $\psi_{0}, c_{1}^{0}, c_{2}^{0}, T^{0}$, we obtain quartic equation in the perturbation decrement in the form

$$
E \lambda^{4}+F \lambda^{3}+G \lambda^{2}+M \lambda+N=0
$$

where $E, F, G, M, N$ depend in a complicated way on $\operatorname{Pr}_{i}$, $\mathrm{Le}_{i}, \mathrm{Ra}_{i}, \mathrm{Ra}, \tau_{i j}$ and modes of perturbation. Let us consider only the monotonic perturbations which decrements are real and the stability boundary (subject to $\lambda=0$ ) is determined by the relation

$$
\begin{gathered}
\frac{k^{6}}{(\pi l)^{2}}=\left(A-\tau_{22}\right) \mathrm{Ra}_{1}+(B-1) \tau_{22} \mathrm{Ra}_{2}+\mathrm{Ra} \\
k^{2}=\pi^{2}\left(n^{2}+m^{2}+l^{2}\right)
\end{gathered}
$$

Eq. (14) in the coordinates $\left(\mathrm{Ra}_{1}, \mathrm{Ra}_{2}, \mathrm{Ra}\right)$ describes the surface dividing the areas of stable diffusion in the nonisothermal conditions and the monotonic perturbations. When $\mathrm{Ra}=0$ (isothermal mixture) then the equilibrium crisis is due to the pure concentration convection [17] and instability is possible in the following case

$$
\left(A-\tau_{22}\right) \mathrm{Ra}_{1}+(B-1) \tau_{22} \operatorname{Ra}_{2} \succ \frac{k^{6}}{(\pi l)^{2}}
$$

Note that depending on the relative position of the mixture's components in the diffusion channel, a sign of the Rayleigh number should be considered in Eqs. (14) and (15).

If $\mathrm{Ra}_{1}=\mathrm{Ra}_{2}=\mathrm{Ra}$, i.e. we have a homogeneous mixture (concentration gradient is absent) then the critical Rayleigh number is determined by the ratio

$$
\mathrm{Ra}_{c r}=\frac{k^{6}}{(\pi l)^{2}}
$$

Considering that diffusion unstable system in which the determining factor is the mutual competition of the concentration gradients against the background of the temperature heterogeneousness has been studied, then specifying the values of Ra in Eq. (14) in the coordinates $\left(\mathrm{Ra}_{1}, \mathrm{Ra}_{2}\right)$ we can obtain the spectrum of the boundary lines that define the process stabilization (destabilization). General analysis on the basis of Eq. (14) enables us to determine the stability and instability areas of diffusion under nonisothermal conditions (see Fig. 6) and to show the stabilizing role of the temperature gradient when heating from above. 


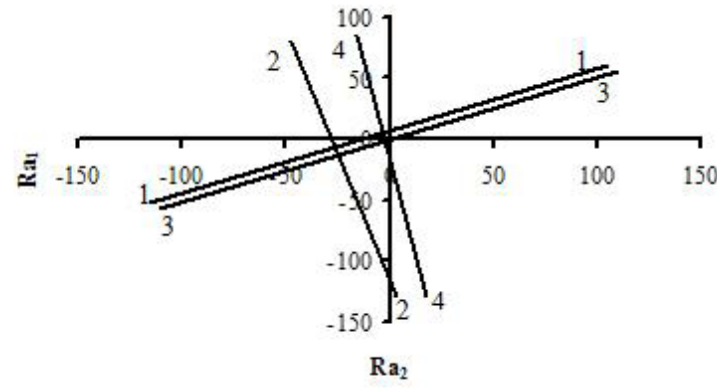

Figure 6. Neutral line of the monotonic instability in the diffusion under nonisothermal conditions for the different values of $\mathrm{Ra}$. $0.8 \mathrm{He}+0.2 \mathrm{Ar}-\mathrm{N}_{2}, 1-1-\mathrm{Ra}=5 ; 3-3-\mathrm{Ra}=20$; $0.4 \mathrm{He}+0.6 \mathrm{Ar}-\mathrm{N}_{2}, 2-2-\mathrm{Ra}=5 ; 4-4-\mathrm{Ra}=20$.

However, for a number of systems including the investigated experimentally one, a reduction of the diffusion Rayleigh number of the first component is due to the concentration of the heavy component (compare $\mathrm{Ra}_{1}$ value at various concentrations shown in Fig. 6) and monotonic disturbances in the system may occur in case of $\mathrm{Ra} \prec \mathrm{Ra}_{c r}$. This effect for binary mixtures was obtained in [7], and further defined as the "buoyancy effect".

\section{Conclusions}

Thus, the study of diffusion unstable systems in the nonisothermal conditions and in the limiting linear consideration (stationary problem and the absence of cross effects) shows the considerable influence of temperature on the intensity of unstable process and the occurrence of new effects related to the thermal concentration mechanism. The intensity of unstable diffusion process in ternary gas mixture $\mathrm{He}+\mathrm{Ar}-\mathrm{N}_{2}$ decreases in the temperature interval $278.0-473.0 \mathrm{~K}$ being at its minimum with the increase of temperature gradient. Then the molecular transfer begins to prevail over the convective one and the process tends to the stabilization. For binary mixtures $\mathrm{N}_{2} \mathrm{O}-\mathrm{CO}_{2}$ and $\mathrm{CO}_{2}-$ $\mathrm{N}_{2} \mathrm{O}$ heated from below critical temperature difference varies fivefold. Heating in the system $0.279 \mathrm{C}_{3} \mathrm{H}_{8}+$ $0.721 \mathrm{CO}_{2}-\mathrm{CO}_{2}$ results in the large rate of convection when the change of convective mass transfer occurs (concentration of passed component has a maximum value due to the temperature difference). In terms of the stability theory, a boundary line is determined, dividing the Rayleigh number plane into the regions of the diffusion and convective mass transfer. The comparison of theoretical results with the experimental data for the study of the effect of temperature difference on the diffusion mixing in the system $\mathrm{He}+\mathrm{Ar}-\mathrm{N}_{2}$ indicates qualitative agreement.

\section{References}

1. M. Sankar, Younghae Do, Int. Comm. Heat Mass Transfer, 37 (2010)

2. S. Chen, J. Tölke, M. Krafczyk, Int. Comm. Heat Mass Transfer, 31 (2010)
3. M. Sankar, Youngyong Park, J.M. Lopez, Younghae Do, Int. Comm. Heat Mass Transfer, 54 (2011)

4. W.A. Khan, A. Aziz, M. Sankar, Younghae Do, Int. Comm. Heat Mass Transfer, 50 (2011)

5. D.D. Joseph, Stability of Fluid Motions (Springer, Berlin, 1976)

6. L.Kh. Ingel, Tech. Phys, 54(2) (2009)

7. G.Z. Gershuni, E.M. Zhukhovitskii, Convective Stability of Incompressible Fluids (Keter, Jerusalem, 1976)

8. L. Miller, E.A. Mason, Phys. Fluids, 9(4) (1966)

9. Yu.I. Zhavrin, V.N. Kosov, J. Eng. Phys., 55(1) (1988)

10. V.D. Seleznev, V.N. Kosov, I.V. Poyarkov, O.V. Fedorenko, M.T. Beketaeva, APhysPolA, 123 (2013)

11. V. Kossov, D. Kulzhanov, I. Poyarkov, O. Fedorenko, MME, 3 (2013)

12. A.G. Shashkov, T.N. Abramenko, Cross-effects in gaseous mixtures (Minsk, Nauka I Tekhnika, 1976)

13. Yu.I. Zhavrin, A.Z. Aitkozhaev, V.N. Kosov, S.A. Krasikov, Technical Physics Letters, 21(3) (1995)

14. Yu.I. Zhavrin, M. Mukamedenkyzy, I.V. Poyarkov, Tech. Phys, 52(7) (2007)

15. V.D. Seleznev, V. G. Smirnov, Zh. Tekh. Fiz., 51 (1981)

16. V.N. Kosov, V.D. Seleznev, Yu.I. Zhavrin, J. Tech. Phys, 42 (1997)

17. Yu.I. Zhavrin, V.N. Kossov, J. Eng. Phys Therm., 60(3) (1991) 